\title{
Neutrino magnetic moments and low-energy solar neutrino-electron scattering experiments
}

\author{
S. Pastor ${ }^{a *}$, J. Segura ${ }^{b \dagger}$, V.B. Semikoz ${ }^{a \ddagger}$ J.W.F. Valle ${ }^{a \S}$ \\ ${ }^{a}$ Instituto de Física Corpuscular - C.S.I.C. \\ Departament de Fúsica Teòrica, Universitat de València \\ 46100 Burjassot, València, SPAIN \\ http://neutrinos.uv.es \\ ${ }^{b}$ Instituto de Bioingeniería \& \\ Departamento de Estadística y Matemática Aplicada \\ Universidad Miguel Hernández, Edificio La Galia \\ 03206 Elche, Alicante, SPAIN
}

\begin{abstract}
The scattering of solar neutrinos on electrons is sensitive to the neutrino magnetic moments through an interference of electromagnetic and weak amplitudes in the cross section. We show that future low-energy solar neutrino experiments with good angular resolution can be sensitive to the resulting az-
\end{abstract}

\footnotetext{
*E-mail: sergio@flamenco.ific.uv.es

${ }^{\dagger}$ E-mail: javier.segura@umh.es

${ }^{\ddagger}$ E-mail: semikoz@izmiran.rssi.ru On leave from Institute of Terrestrial Magnetism, the Ionosphere and Radio Wave Propagation of the Russian Academy of Sciences, IZMIRAN, Troitsk, Moscow region, 142092, Russia.

$\S$ E-mail valle@flamenco.ific.uv.es
} 
imuthal asymmetries in event number and should provide useful information on non-standard neutrino properties such as magnetic moments. We compare asymmetries expected at Hellaz (mainly pp neutrinos) with those at the Kamiokande and Super-Kamiokande experiments (Boron neutrinos), both for the case of Dirac and Majorana neutrinos and discuss the advantages of low energy experiments. Potentially interesting information on the solar magnetic fields may be accessible. 


\section{INTRODUCTION}

Most non-standard properties of neutrinos arise from non-zero masses [1]:2]. Among these electro-magnetic dipole moments play an important role [3]. Here we are concerned with a particular effect in neutrino-electron scattering for neutrinos from the Sun which possess a Dirac magnetic moment [4] or transition magnetic moments [5] in the case of Majorana neutrinos. The latter is especially interesting first of all because it is more fundamental theoretically, and because Majorana neutrinos are the ones which arise in most extensions of the Standard Model. Moreover, the effects of Majorana transition moments can be resonantly enhanced when neutrinos propagate in media [6] such as the Sun, providing one of the attractive solutions to the solar neutrino problem [7]. Another practical advantage in favour of Majorana transition moments is that, in contrast to Dirac-type magnetic moments, these are substantially less stringently constrained by astrophysics [B]

For pure left-handed neutrinos the weak interaction and the electro-magnetic interaction amplitudes on electrons do not interfere, since the weak interaction preserves neutrino helicity while the electro-magnetic does not. As a result the cross section depends quadratically on $\mu_{\nu}$.

However, if there exists a process capable of converting part of the initially fully polarized $\nu_{e}$ 's, then an interference term arises, proportional to $\mu_{\nu}$, as pointed out e.g. in ref. [9]. This term depends on the angle between the component of the neutrino spin transverse to its momentum and the momentum of the outgoing recoil electron. Therefore the event count rates expected in an experiment would exhibit an asymmetry with respect to the above defined angle. Such asymmetry would not show up in earth-bound laboratory experiments even with stronger magnetic fields, since the helicity-flip could be caused only by the presence of a neutrino mass and is therefore negligible [10]. However, in the solar convective zone one may find a magnetic field extended over a tenth or so of the solar radius and, most importantly, the neutrino depolarization could be resonant in the Sun. Even if the Sun possesses only a relatively modest large-scale magnetic field $B_{\perp} \sim 10^{4} \mathrm{G}$ in the convective 
region $\left(L \sim L_{\text {conv }} \simeq 3 \times 10^{10} \mathrm{~cm}\right)$, and for a neutrino magnetic moment of the order $10^{-11} \mu_{B}$ such a spin-flip process may take place with sizeable rates, since in such a case one has $\mu_{\nu} B_{\perp} L \sim 1$.

Barbieri and Fiorentini considered [9] the conversions $\nu_{e L} \rightarrow \nu_{e R}$ in the Sun as a result of the spin-flip by a toroidal magnetic field in the convective zone. They showed that the azimuthal asymmetry could be observable in a real time solar ${ }^{8} \mathrm{~B}$-neutrino experiment and as large as $20 \%$ for an electron kinetic energy threshold of $W_{e}=5 \mathrm{MeV}$. They chose a fixed $\nu_{e}$ survival probability $P_{e}=1 / 3$ (as suggested at that time by the Homestake experiment) and the maximal Dirac magnetic moment allowed by laboratory experiments, $\mu_{\nu} \simeq 10^{-10} \mu_{B}$.

On the other hand, Vogel and Engel [11] emphasized that if an asymmetry in the scattering of solar neutrinos exists, recoil electrons will be emitted copiously along the direction of the neutrino polarization in the plane orthogonal to the neutrino momentum. They calculated the asymmetry expected for solar ${ }^{8} \mathrm{~B}$ neutrinos with $\mu_{\nu}=10^{-10} \mu_{B}$ and concluded that it would be difficult to detect because of the poor angular resolution of the experiments. Moreover, as we will see later, both [9] and [11] overestimated the asymmetry. Thus their calculations are not accurate.

In this paper we correct results for the asymmetry in the case presented by [9] and [11] for high energy ${ }^{8} \mathrm{~B}$ neutrinos. In addition we compare them with the expected asymmetry in the case of a Majorana transition magnetic moment of the same magnitude. More importantly, we show the sensitivity of planned solar neutrino experiments in the low energy region $(\omega \lesssim 1 \mathrm{MeV})$ to the azimuthal asymmetries that are expected in the recoil electron event rates, arising from the above electro-weak interference term. We calculate the asymmetry for the low-energy $p p$-neutrinos fixing the survival probability at $P_{e}=0.5$. This gives the maximum expected asymmetry and seems phenomenologically reasonable in order to convert the initial solar $\nu_{e L}$ 's via the Resonant Spin-Flavour Precession (RSFP) scenario. In particular we calculate the asymmetry that could be observed in the azimuthal distribution of events in an experiment like the proposed Hellaz [12], sensitive to the fundamental $p p$ neutrinos from the Sun. The Multi-Wire-Chamber in Hellaz should measure both the recoil 
electron energy $T$ and the recoil electron scattering angle $\theta$ with good precision. Moreover Hellaz should be sensitive to the azimuthal angle $\phi$, measuring the number of events in $\phi$-bins. We discuss the sensitivity of the Hellaz experiment for probing $\mu_{\nu}$ and compare it with planned accelerator experiments. In particular there are very interesting new projects, such as the future ITEP-Minnesota experiment, where they plan to search $\mu_{\nu} / \mu_{B}$ down to $3 \cdot 10^{-11}$ with reactor anti-neutrinos [13], and the LAMA experiment, which will use a powerful isotope neutrino source [14].

Finally, we also refine our calculations of the azimuthal asymmetry expected for $p p$ neutrinos at Hellaz using a realistic energy-dependent conversion probability $P_{e}$ based on a simple model for resonant spin flip conversions in the Sun.

\section{NEUTRINO-ELECTRON SCATTERING CROSS SECTIONS}

We consider the neutrino-electron scattering process $\nu_{e}\left(k_{1}\right)+e\left(p_{1}\right) \rightarrow \nu_{e}\left(k_{2}\right)+e\left(p_{2}\right)$ when the initial flux of neutrinos is not completely polarized, as a result of conversions induced by non-zero transition magnetic moments in the Sun. In other words, our flux is a mixture of both the original left-handed solar neutrinos with the converted ones (right-handed). We consider both conventional Dirac-type magnetic moments as well as Majorana transition moments. The differential cross section can be written as a sum of three terms, (for details, see for instance [15])

$$
\frac{d \sigma}{d T d \phi}=\left(\frac{d \sigma}{d T d \phi}\right)_{w e a k}+\left(\frac{d \sigma}{d T d \phi}\right)_{e m}+\left(\frac{d \sigma}{d T d \phi}\right)_{i n t},
$$

where $T$ is the recoil energy of electrons and $\phi$ the azimuthal angle defined in figure 11.

Let us first assume that only the Dirac $\nu_{e}$ magnetic moment exists, $\mu_{\nu_{e}}$. For ultrarelativistic neutrinos the expressions for the weak and electro-magnetic terms are the following

$$
\left(\frac{d \sigma}{d T d \phi}\right)_{\text {weak }}=P_{e} \frac{G_{F}^{2} m_{e}}{\pi^{2}}\left[g_{e L}^{2}+g_{R}^{2}\left(1-\frac{T}{\omega}\right)^{2}-\frac{m_{e} T}{\omega^{2}} g_{e L} g_{R}\right]
$$




$$
\left(\frac{d \sigma}{d T d \phi}\right)_{e m}=\frac{\alpha^{2}}{2 m_{e}^{2}}\left(\frac{\mu_{\nu_{e}}}{\mu_{B}}\right)^{2}\left[\frac{1}{T}-\frac{1}{\omega}\right]
$$

Here $P_{e}$ is the survival probability of the initial $\nu_{e L}, \omega$ is the incoming neutrino energy, and $g_{e L}=\sin ^{2} \theta_{W}+0.5$ and $g_{R}=\sin ^{2} \theta_{W}$ are the constants of the Standard Model with $\sin ^{2} \theta_{W} \simeq 0.23$

In such a case there is an interference term proportional to $\mu_{\nu}$ [9],

$$
\left(\frac{d \sigma}{d T d \phi}\right)_{i n t}=-\frac{\alpha G_{F}}{2 \sqrt{2} \pi m_{e} T}\left(\frac{\mu_{\nu_{e}}}{\mu_{B}}\right) \vec{p}_{2} \cdot \vec{A}_{D}(T, \omega)
$$

where

$$
\vec{A}_{D}(T, \omega) \equiv\left[g_{e L}+g_{R}\left(1-\frac{T}{\omega}\right)\right] \vec{\xi}_{\perp}
$$

Here $\vec{\xi}_{\perp}$ is the transverse component of the neutrino polarization spin vector with respect to its momentum. It is a function of the $\nu_{e L}$ survival probability, $\left|\vec{\xi}_{\perp}\right|=2 \sqrt{P_{e}\left(1-P_{e}\right)}$. This interference term depends on the angle between $\vec{\xi}_{\perp}$ and the momentum of the outgoing electron $\vec{p}_{2}$. Eq. (4) may be written as a function of the azimuthal angle $\phi$ (as defined in figure 回) using

$$
\vec{p}_{2} \cdot \vec{\xi}_{\perp}=\left|\vec{p}_{2}\right| \sin \theta\left|\vec{\xi}_{\perp}\right| \cos \phi=\sqrt{2 m_{e} T\left(1-\frac{T}{T_{\max }}\right)}\left|\vec{\xi}_{\perp}\right| \cos \phi
$$

where $T_{\max }=2 \omega^{2} /\left(m_{e}+2 \omega\right)$ is the maximum electron recoil energy.

Let us explain here why the results of [9] and [11] for Dirac neutrinos are not correct. First, the weak term in eq. (11a) of Barbieri \& Fiorentini is a factor 2 less than our eq. (2), while the interference term coincides with ours. On the other hand Vogel \& Engel used an interference term (their eq. (A9)) which is a factor 2 bigger than our eq. (田). Thus both overestimated the asymmetry by an approximate factor two. This also agrees with a recent calculation in reference [16].

If neutrinos are Majorana particles they can only possess a transition magnetic moment $\mu_{12}$. For simplicity we assume the case of CP conservation. For definiteness, moreover, we consider the case of two neutrino species, $\nu_{e}$ and $\nu_{\mu}$, with positive relative CP-parity [5]. 
The three terms of the differential cross section will include an electro-magnetic term (same as eq. (3) with $\mu \rightarrow \mu_{12}$ ), a weak term,

$$
\begin{aligned}
& \left(\frac{d \sigma}{d T d \phi}\right)_{\text {weak }}=\frac{G_{F}^{2} m_{e}}{\pi^{2}}\left\{P_{e}\left[g_{e L}^{2}+g_{R}^{2}\left(1-\frac{T}{\omega}\right)^{2}-\frac{m_{e} T}{\omega^{2}} g_{e L} g_{R}\right]+\right. \\
& \left.+\left(1-P_{e}\right)\left[g_{R}^{2}+g_{\mu L}^{2}\left(1-\frac{T}{\omega}\right)^{2}-\frac{m_{e} T}{\omega^{2}} g_{\mu L} g_{R}\right]\right\}
\end{aligned}
$$

where $g_{\mu L}=\sin ^{2} \theta_{W}-0.5$, and an interference term

$$
\left(\frac{d \sigma}{d T d \phi}\right)_{i n t}=-\frac{\alpha G_{F}}{4 \sqrt{2} \pi m_{e} T}\left(\frac{\mu_{12}}{\mu_{B}}\right) \vec{p}_{2} \cdot \vec{A}_{M}(T, \omega)
$$

where we have defined

$$
\vec{A}_{M}(T, \omega) \equiv\left[\left(g_{e L}+g_{\mu L}+2 g_{R}\right)\left(2-\frac{T}{\omega}\right)+\left(g_{e L}-g_{\mu L}\right) \frac{T}{\omega}\right] \vec{\xi}_{\perp}^{e \bar{\mu}}
$$

Here the mixed polarization vector is given by [15]

$$
\left|\vec{\xi}_{\perp}^{e \bar{\mu}}\right|=2 \sqrt{P_{e} P_{\bar{\mu}}}
$$

where $P_{\bar{\mu}}=1-P_{e}$ is the $\nu_{e L} \rightarrow \bar{\nu}_{\mu R}$ conversion probability.

\section{EXPECTED AZIMUTHAL ASYMMETRIES IN HELLAZ}

The relevant quantity to be measured in neutrino-electron scattering experiments capable of measuring directionality of the outgoing $e^{-}$(like Hellaz) is the event number azimuthal distribution, namely

$$
\frac{d N}{d \phi}=N_{e} \sum_{i} \Phi_{0 i} \int_{T_{T h}}^{T_{\max }} d T \int_{\omega_{\min }(T)}^{\omega_{\max }} d \omega \lambda_{i}(\omega) \epsilon(\omega) \frac{d \sigma}{d T d \phi}(\omega, T)
$$

where $d \sigma / d T d \phi$ is given in eq. (1),$\epsilon(\omega)$ is the efficiency of the detector (which we take as unity for energies above the threshold, for simplicity), and $N_{e}$ is the number of electrons in the fiducial volume of the detector. The sum in the above equation is done over the solar neutrino spectrum, where $i$ corresponds to the different reactions $i=p p,{ }^{7} \mathrm{Be}, p e p,{ }^{8} \mathrm{~B} \ldots$, characterized by a differential spectrum $\lambda_{i}(\omega)$ and an integral flux $\Phi_{0 i}$. 
In the previous section we found that the azimuthal distribution of the number of events can be written as

$$
\frac{d N}{d \phi}=n_{w e a k}+n_{e m}+n_{\text {int }} \cos \phi
$$

where $n_{\text {weak }}\left(n_{\text {em }}\right)$ accounts for the weak (electro-magnetic) contributions, while $n_{\text {int }}$ is the interference term.

The differential azimuthal asymmetry is defined as

$$
\left.\frac{d A}{d \phi}\right|_{\phi^{\prime}}=\frac{\left.\frac{d N}{d \phi}\right|_{\phi^{\prime}}-\left.\frac{d N}{d \phi}\right|_{\phi^{\prime}+\pi}}{\left.\frac{d N}{d \phi}\right|_{\phi^{\prime}}+\left.\frac{d N}{d \phi}\right|_{\phi^{\prime}+\pi}}=\frac{n_{\text {int }}}{n_{\text {weak }}+n_{e m}} \cos \phi^{\prime}
$$

where $\phi\left(\phi^{\prime}\right)$ is measured with respect to the direction of the magnetic field $\vec{B}_{\odot}$, which we will assume to be along the positive $x$-axis (see fig. 1).

One can also define an integrated (over $\phi$ ) asymmetry

$$
\mathcal{A}\left(\phi^{\prime}\right)=\frac{N_{2}\left(\phi^{\prime}\right)-N_{1}\left(\phi^{\prime}\right)}{N_{2}\left(\phi^{\prime}\right)+N_{1}\left(\phi^{\prime}\right)},
$$

where

$$
\begin{aligned}
& N_{2}\left(\phi^{\prime}\right)=\int_{\phi^{\prime}}^{\phi^{\prime}+\pi} \frac{d N}{d \phi} d \phi=\pi\left(n_{\text {weak }}+n_{\text {em }}\right)-2 n_{\text {int }} \sin \phi^{\prime} \\
& N_{1}\left(\phi^{\prime}\right)=\int_{\phi^{\prime}+\pi}^{\phi^{\prime}+2 \pi} \frac{d N}{d \phi} d \phi=\pi\left(n_{\text {weak }}+n_{\text {em }}\right)+2 n_{\text {int }} \sin \phi^{\prime}
\end{aligned}
$$

and then one gets

$$
\mathcal{A}\left(\phi^{\prime}\right)=-\frac{2 n_{\text {int }}}{\pi\left(n_{\text {weak }}+n_{\text {em }}\right)} \sin \phi^{\prime}
$$

which is directly related to the differential asymmetry by

$$
\mathcal{A}\left(\phi^{\prime}\right)=-\left.\frac{2}{\pi} \frac{d A}{d \phi}\right|_{\phi^{\prime}} \tan \phi^{\prime}
$$

Let us define the maximum integrated asymmetry measurable by the experiment, 


$$
A=\frac{2 n_{\text {int }}}{\pi\left(n_{\text {weak }}+n_{\text {em }}\right)}
$$

Then

$$
\mathcal{A}\left(\phi^{\prime}\right)=-A \sin \phi^{\prime}
$$

where $A$ is manifestly positive.

It is important to emphasize that Hellaz will be the first experiment which is potentially sensitive to azimuthal asymmetries since the directionality of the outgoing $e^{-}$can be measured. The angular resolution is expected to be $\Delta \theta \sim \Delta \phi \sim 30 \operatorname{mrad} \sim 2^{\circ}$, substantially better than that of Super-Kamiokande. Notice also that the width of the Cerenkov cone defined by the angle $\theta$ is very narrow for high-energy boron neutrinos, as one can see from eq. (6). In contrast, for $p p$ neutrino energies accessible at Hellaz $\left(T_{\max } \simeq 0.26 \mathrm{MeV}\right.$, $\left.T_{t h} \simeq 0.1 \mathrm{MeV}\right)$ we estimate that $\theta$ can be as large as $48^{\circ}$.

Let us now discuss how the measurement of the azimuthal asymmetry could be carried out considering that $\vec{B}_{\odot}$ is constant over a given period of time but its direction is unknown. One should collect events in every $\phi$-bin, where $\phi$ is defined with respect to some arbitrarily chosen axis, and then take for different $\phi^{\prime} s$ the ratio $\mathcal{A}\left(\phi^{\prime}\right)$ which should show a $\sin \phi^{\prime}$ dependence with a maximum equal to $A$. This maximum will show us the angle $\phi_{0}$ which corresponds to the direction of $\vec{B}_{\odot}\left(\phi_{0}=0\right.$ if $\vec{B}_{\odot}$ goes along the positive x axis). Then the direction of $\vec{B}_{\odot}$ is measured together with $A$. Since this direction may change in time the experiment should accumulate events until the maximum $\sin \phi$-like correlation in $\mathcal{A}(\phi)$ is found and then start a new event counting period when such correlation goes away due to the changing direction of $\vec{B}_{\odot}$. Therefore the value of $A$ could be extracted by performing a series of such measurements.

We have calculated the maximal integrated azimuthal asymmetry $A$ for the case of ${ }^{8} \mathrm{~B}$ neutrinos, i.e. for the situation described in references [9] and [11]. In order to do this we have made use of the corresponding differential ${ }^{8} \mathrm{~B}$ neutrino spectrum in the Standard Solar Model [17]. Our results are shown in figure 2. One can see that the asymmetry in the Dirac 
diagonal case $\left(\nu_{e L} \rightarrow \nu_{e R}\right)$ is in fact approximately a factor two smaller than predicted by Barbieri \& Fiorentini. The asymmetry in the Majorana case $\left(\nu_{e L} \rightarrow \bar{\nu}_{\mu R}\right)$ for the equivalent $\mu$ value is, as expected, smaller since there are two active species in the neutrino flux so that the weak term (which enters in the denominator in eq. (17)) becomes larger.

Figure 3 shows the results obtained by a similar analysis for the case of $p p$-neutrinos, again taking into account the theoretically predicted differential $p p$ neutrino spectrum and a more realistic value of the survival probability for the RSFP scenario in the $\operatorname{Sun}\left(P_{e}=0.5\right)$. Searching for such an asymmetry would be quite an interesting physics task in an Hellaz-like experiment.

The dependence of $A$ on the value of the magnetic moment deserves a more detailed analysis. One can write

$$
A=\frac{\mu_{\nu} a_{i n t}}{a_{\text {weak }}+\mu_{\nu}^{2} a_{e m}}
$$

with $\mu_{\nu} a_{\text {int }}=4 n_{\text {int }}, a_{\text {weak }}=2 \pi n_{\text {weak }} \equiv N_{\text {weak }}$ and $\mu_{\nu}^{2} a_{e m}=2 \pi n_{e m} \equiv N_{e m}$. It follows that $A$ is maximized for $N_{\text {weak }}=N_{\text {em }}$, e.g. when the pure weak term is equal to the electro-magnetic contribution. This fact favours $p p$-neutrinos with respect to high energy neutrinos, since such a maximum is reached for lower $\mu_{\nu}$ values precisely due to the lower energies considered. In fact, for an energy threshold of recoil electrons $W_{e}=0.1 \mathrm{MeV}$ (reachable at Hellaz) the maximal asymmetry is reached for $\mu_{\nu} \simeq 3 \cdot 10^{-11} \mu_{B}$ in contrast to Boron neutrinos, for which the maximal asymmetry is reached for $\mu_{\nu} \simeq 10^{-10} \mu_{B}$. This way one sees that figs. 2 and 3 describe approximately the most favourable situation for measuring $A$ (see also figure $\mathbb{1}$ ).

The fact that $d A / d \mu_{\nu}=0$ is reached when the number of weak events is equal to the number of electro-magnetic events seems to suggest that the measurement of the total number of events $N_{\text {weak }}+N_{\text {em }}$ would be enough to rule out the values of $\mu_{\nu}$ to which the asymmetry is sensitive. However, it is not so if one bears in mind that:

1. background events from other processes always increase the total number of events. Thus one needs to perform a good subtraction of background events to get some information on the $\mu_{\nu}$ term. On the other hand, assuming that the background is 
isotropic in the azimuthal plane, it should not be present in the numerator of $A$ and then if some asymmetry is measured one can be confident that it is due to a neutrino magnetic moment;

2. the asymmetry is a ratio of event numbers. Thus global normalization uncertainties (e.g. in the total neutrino fluxes) completely drop out from the asymmetry. On the other hand energy-dependent uncertainties (in the neutrino spectrum or detection efficiencies) will be reduced since the same integrations appear in the numerator and in the denominator;

Last, but not least, if resonant spin flavour precessions really take place, one can not use the standard weak cross section and subtract it in order to get information on $\mu_{\nu}$; the signal becomes then uncertain since the "weak background" becomes unknown.

For all these reasons the asymmetry measurement is preferred. It suffers, of course from the dependence on the magnetic field direction, which is unknown. However, sensitivity to that information is also of potential astrophysical interest.

\section{A SIMPLE MODEL FOR THE CONVERSION PROBABILITY}

We now consider how the above results are affected by the energy dependence expected in the conversion probability in the RSFP scenario. The general evolution Hamiltonian characterizing the system of two Majorana neutrinos with a non-zero transition moment is four-dimensional [5]. For simplicity we illustrate the energy dependence of the conversion probability in the simplest realization of the RSFP scenario [6] where vacuum mixing is neglected. In this case the Hamiltonian becomes $2 \times 2$ and the conversion probability $P\left(\nu_{e L} \rightarrow \bar{\nu}_{\mu R}\right)$ is given analytically as (see for instance [18])

$$
P\left(\nu_{e} \rightarrow \bar{\nu}_{\mu}\right)=\frac{1}{2}-\left(\frac{1}{2}-P_{L Z}\right) \cos 2 \theta_{m}
$$

Here $\theta_{m}$ is the effective neutrino mixing angle in matter 


$$
\tan 2 \theta_{m}=\frac{2 \mu_{12} B_{\odot}}{\frac{\Delta m^{2}}{2 \omega}-2 V_{N}-V_{C}}
$$

where $\mu_{12}$ denotes the transition magnetic moment, $\Delta m^{2}=m_{\nu_{\mu}}^{2}-m_{\nu_{e}}^{2}$ and $V_{C}\left(V_{N}\right)$ is the effective potential of the neutrinos in the medium that arises due to charged (neutral) current interactions. On the other hand $P_{L Z}$ is the Landau-Zener transition probability,

$$
P_{L Z}=\exp \left(-\frac{\pi}{4} Q\right) \quad \text { where } \quad Q \simeq \frac{16 \omega\left(\mu_{12} B_{\odot}\right)^{2} 0.1 R_{\odot}}{\Delta m^{2}}
$$

From analyses of experimental data and different models of the solar magnetic field [7], one finds that the data are well reproduced for $\Delta m^{2} \simeq 4 \cdot 10^{-9}-2 \cdot 10^{-8} \mathrm{eV}^{2}$, with a maximum $B_{\odot}$ strength of $25-50 \mathrm{kG}$, assuming $\mu_{12}=10^{-11} \mu_{B}$.

For low-energy solar neutrinos, such as $p p$ neutrinos, the conversion in eq. (21) corresponds to the non-adiabatic regime $Q \lesssim 1$, so that

$$
P\left(\nu_{e L} \rightarrow \bar{\nu}_{\mu R}\right) \simeq 1-P_{L Z}(\omega)
$$

In fig. 5 we show our results for the azimuthal asymmetry of events when the conversion probability depends on the neutrino energy as in eq. (24). We present three choices of $\Delta m^{2}, B_{\odot}$ values that lead to a conversion probability of the order of $40-60 \%$. The case of constant $P_{e}=0.5$ is shown for comparison. One can see from the figure that the dependence of $P_{e}$ on the neutrino energy leads to somewhat smaller azimuthal asymmetries, but qualitatively very similar to those obtained in the previous case, where the energy dependence was neglected. There is basically a compensation of the effect when integrating over energies with the $\nu_{p p}$ spectrum, in such a way that this dependence is smoothed.

\section{SUMMARY AND DISCUSSION}

Measuring azimuthal asymmetries in future low-energy solar neutrino-electron scattering experiments with good angular resolution should be a feasible and illuminating task. Such asymmetries should provide useful information on non-standard neutrino properties such as magnetic moments, as well as on solar magnetic fields. The effect follows from an 
interference of electro-magnetic and weak amplitudes in the cross section. We have seen that low-energy experiments such as Hellaz (sensitive mainly to pp neutrinos) should provide a much better means for the study of azimuthal asymmetries than accessible at the Kamiokande or Super-Kamiokande experiments (sensitive to Boron neutrinos). For equal values of the magnetic moments, the expected asymmetries are larger for Dirac neutrinos than for Majorana neutrino transition moments. However, the Dirac neutrino case is probably less likely, as there is no resonant conversion in the Sun. One exception would be the case of Dirac neutrinos in the presence of twisting magnetic fields [19]. However, although in this case resonant conversions in matter can take place one expects (as mentioned in section 3) a washing out of the asymmetry effect due to the changing magnetic field direction. Therefore the RSFP scenario remains as the most promising possibility. It is also the most interesting one theoretically, since Majorana neutrinos are more fundamental and arise in most models of particle physics beyond the Standard Model.

Note that the discussion given above we have assumed $\nu_{e}$ magnetic moments of the order $10^{-11} \mu_{B}$ which is consistent with present laboratory experiments. Apart from possible effects in red giants, a $\nu_{e}$ transition moment of $10^{-11} \mu_{B}$ is compatible with astrophysical limits, given the present uncertainties in these considerations.

\section{ACKNOWLEDGEMENTS}

The authors thank Tom Ypsilantis for fruitful discussions on the Hellaz experiment. This work has been supported by DGICYT under Grants PB95-1077 and SAB95-506 (V.B.S.), by the TMR network grant ERBFMRXCT960090 and by INTAS grant 96-0659 of the European Union. S.P. was supported by Conselleria d'Educació i Ciència of Generalitat Valenciana. V.B.S. also acknowledges the support of RFFR through grant 97-02-16501. 


\section{REFERENCES}

[1] See, e.g. J.W.F. Valle, Gauge Theories and the Physics of Neutrino Mass, Prog. Part. Nucl. Phys. 26, 91 (1991)

[2] For recent reviews on the status of neutrino mass see by A.Yu. Smirnov, at 28th International Conference on High energy physics, July 1996, Warsaw, Poland, hep-ph/9611465; and by P. Vogel and J.W.F. Valle at WIN97, Capri, Italy, June 1997, Nucl. Phys. B (Proc. Suppl.) 66, 131 (1998), 141

[3] For a discussion of different models for the neutrino magnetic moment $\mu_{\nu}$ see, for instance R.N. Mohapatra and P.B. Pal, Massive Neutrinos in Physics and Astrophysics (World Scientific, 1991)

[4] M.B. Voloshin and M.I. Vysotsky, Sov. J. Nucl. Phys. 44, 845 (1986);

M.B. Voloshin, M.I. Vysotsky and L.B. Okun, Sov. Phys. JETP 64, 446 (1986)

[5] J. Schechter and J.W.F. Valle, Phys. Rev. D24, 1883 (1981), err. Phys. Rev. D25, $283(1982)$

[6] C.S. Lim and W. Marciano, Phys. Rev. D37, 1368 (1988);

E.Kh. Akhmedov, Phys. Lett. B213, 64 (1988)

[7] E.Kh. Akhmedov, The neutrino magnetic moment and time variations of the solar neutrino flux, Preprint IC/97/49, hep-ph/9705451, invited talk given at the 4th International Solar Neutrino Conference, Heidelberg, Germany, April 1997

[8] G. Raffelt, Stars as laboratories for fundamental physics, (Univ. of Chicago Press, Chicago-London 1996)

[9] R. Barbieri and G. Fiorentini, Nucl. Phys. B304, 909 (1988)

[10] See, e.g. W. Grimus, P. Stockinger, Phys. Rev. D57, 1762 (1998)

[11] P. Vogel and J. Engel, Phys. Rev. D39, 3378 (1989) 
[12] F. Arzarello et al., Preprint CERN-LAA/94-19, College de France LPC/94-28 (1994); J. Seguinot et al., Preprint LPC 95 08, College De France, Laboratoire de Physique Corpusculaire (1995)

[13] A.G. Beda, E.V. Demidova, A.S. Starostin and M.B. Voloshin, On the possibility of Low-Background Ge-NaI Spectrometer for Neutrino Magnetic Moment Measurement, Preprint TPI-MINN-97/15-T, UMN-TH-1544-97, May 1997

[14] I.R. Barabanov et al., Searching for a neutrino magnetic moment deeply underground (LAMA project). Talk given by R. Bernabei, WIN97, Capri, June 1997, Nucl. Phys. B (Proc. Suppl.) 66, 222 (1998)

[15] V.B. Semikoz, Nucl. Phys. B498, 39 (1997)

[16] I.V. Gaidaenko, Yad. Fiz. 61, 125 (1998)

[17] See e.g. J.N. Bahcall, Nucl. Phys. B (Proc. Suppl.) 48, 309 (1996)

[18] C.W. Kim and A. Pevsner, Neutrinos in Physics and Astrophysics, (Ed. Harwood Academic Publishers, 1993)

[19] E.Kh. Akhmedov, S.T. Petcov and A.Yu. Smirnov, Phys. Rev. D48, 2167 (1993); Phys. Lett. B309, 95 (1993) 


\section{FIGURES}

FIG. 1. Coordinate system conventions.

FIG. 2. Maximal integrated azimuthal asymmetry $A$ for Boron neutrinos as a function of the electron recoil energy threshold $W_{e}$. Solid line: Dirac case $\left(\nu_{e L} \rightarrow \nu_{e R}\right)$. Dashed line: Majorana case $\left(\nu_{e} \rightarrow \bar{\nu}_{\mu}\right)$. Here $P_{e}$ is the electron neutrino survival probability.

FIG. 3. Maximal integrated azimuthal asymmetry $A$ for $p p$ neutrinos as a function of $W_{e}$. Solid line: Dirac case. Dashed line: Majorana case.

FIG. 4. Dependence of the maximal azimuthal symmetry A on the magnetic moment. Solid line: Dirac case. Dashed line: Majorana case. The threshold energy for recoil electrons is fixed at $W_{e}=0.1 \mathrm{MeV}$ (upper lines) and $W_{e}=0.05 \mathrm{MeV}$ (lower ones).

FIG. 5. The three lower curves show the maximal azimuthal asymmetry $A$ in the simple RSFP model described in section 4 for the Majorana transition moment case for three different choices

of parameters. The upper one gives the expected asymmetry expected for the case of constant $P_{e}=0.5$ (maximum of asymmetry). 


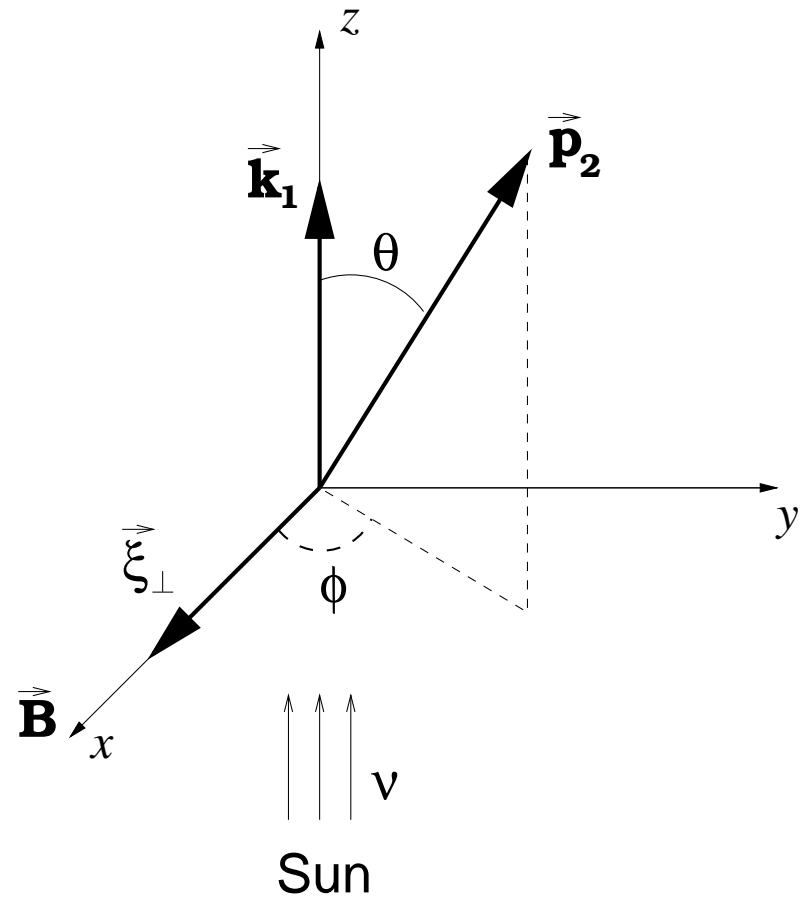

Fig. 1 


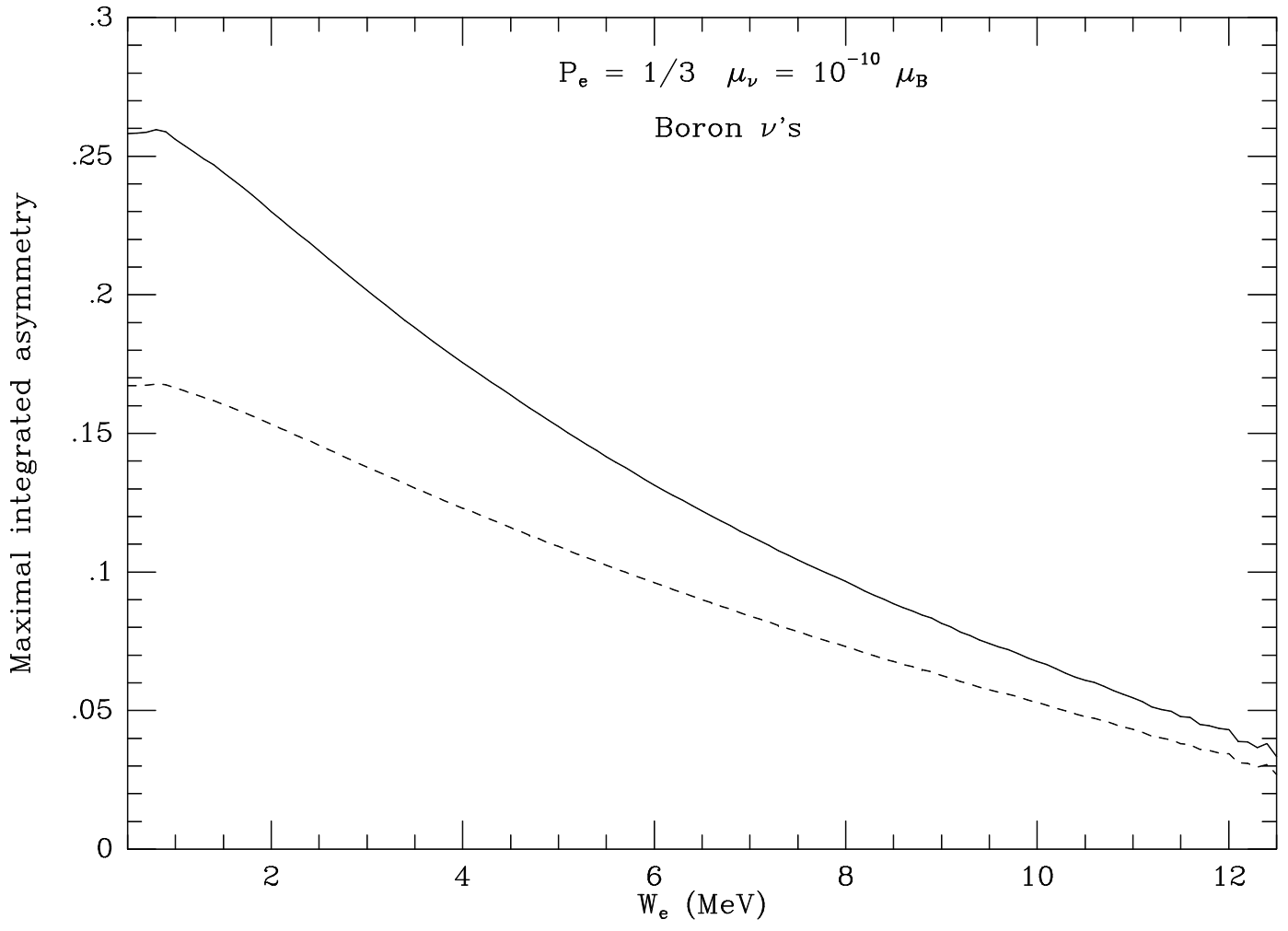

Fig. 2 


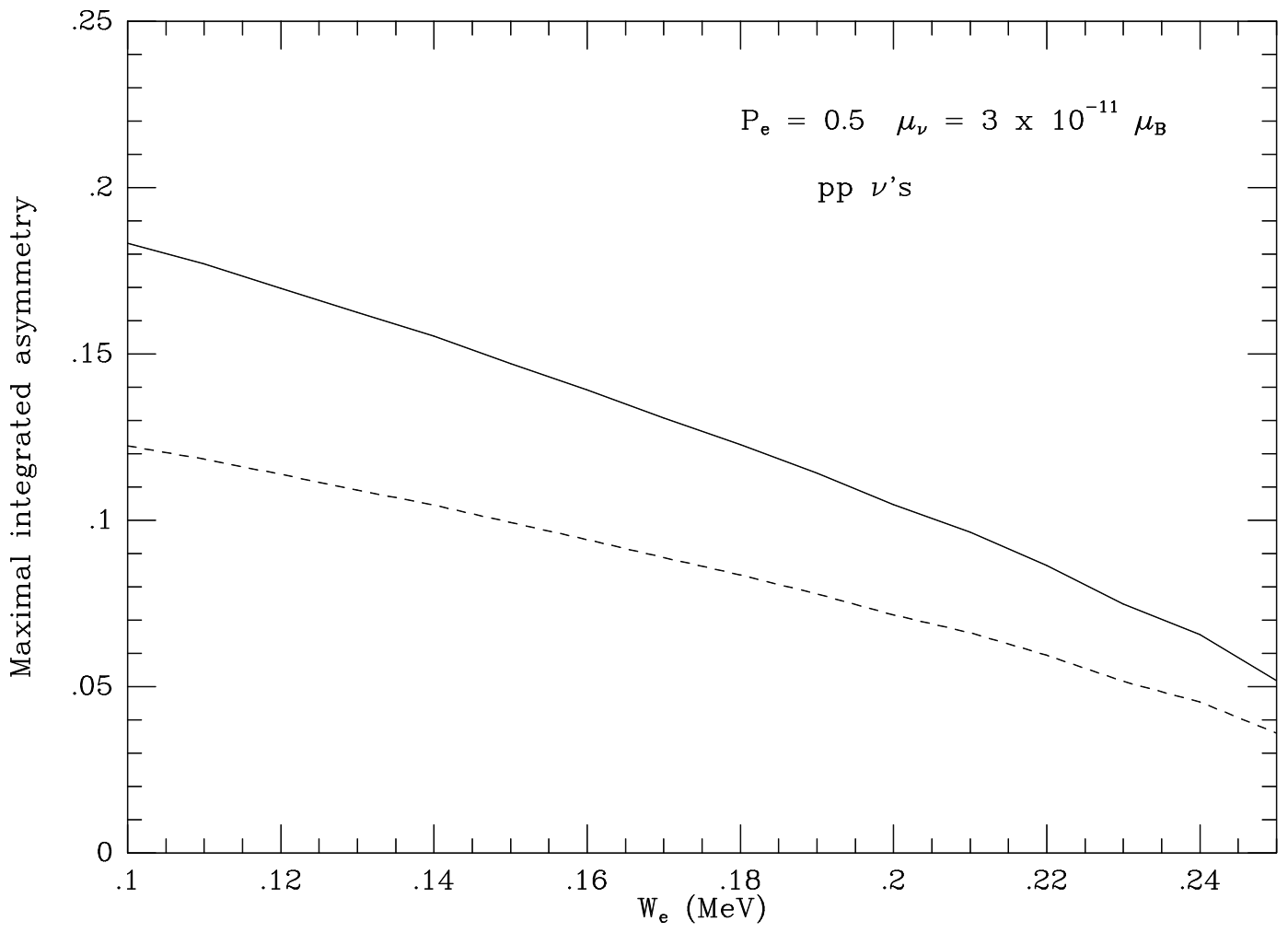

Fig. 3 


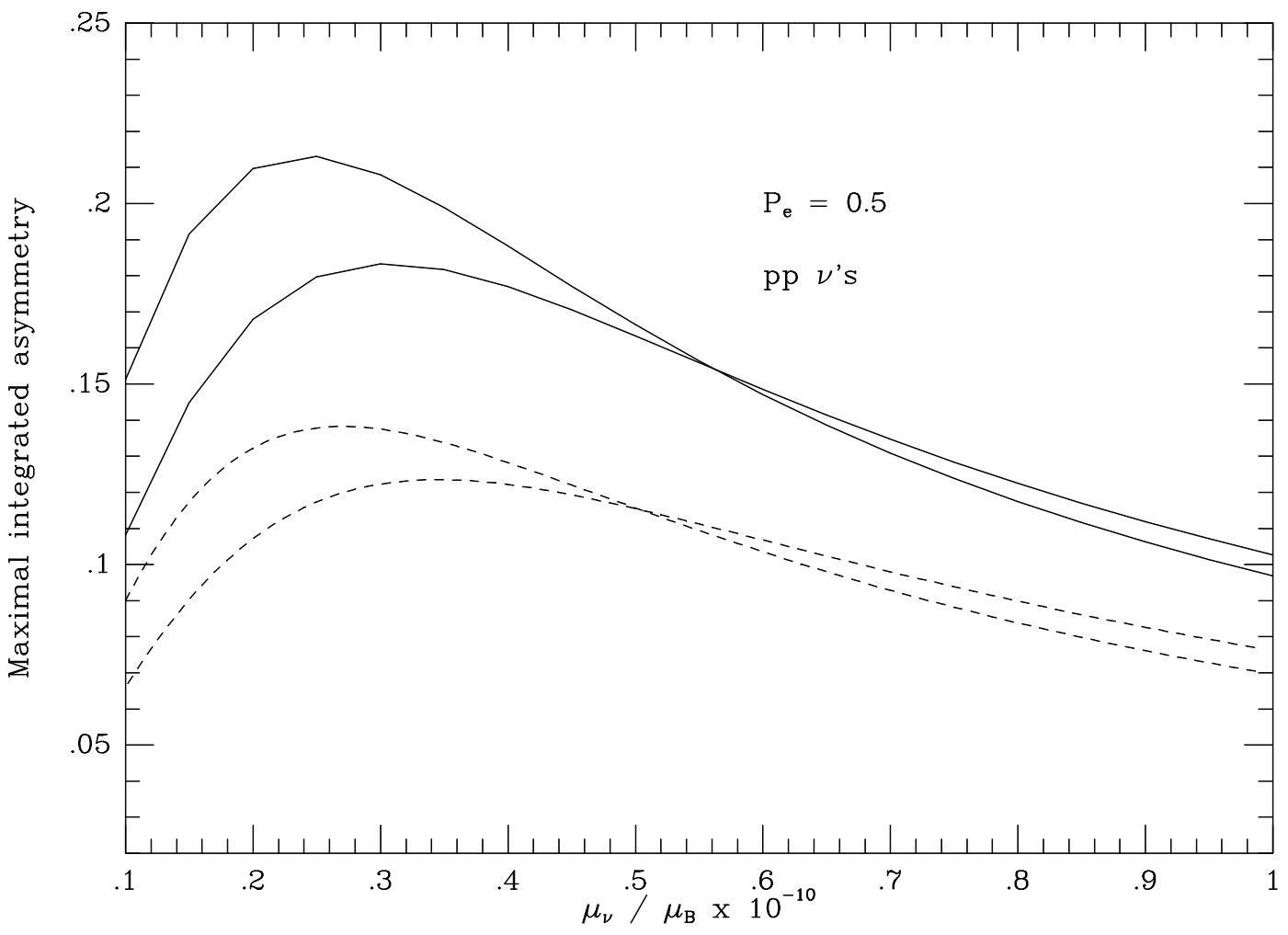

Fig. 4 


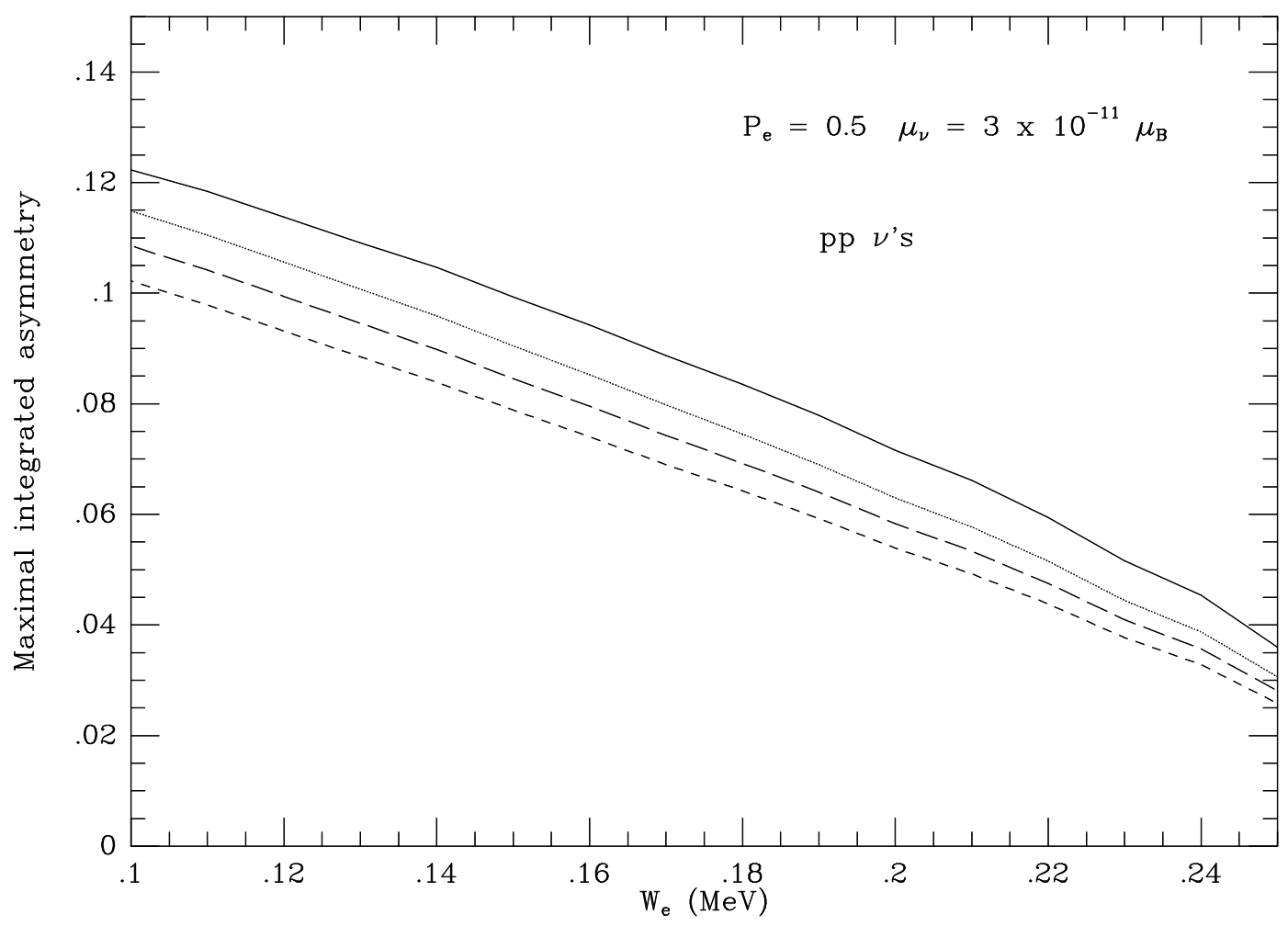

Fig. 5 